\title{
Topogrrafia da córnea após perfuração ocular
}

\author{
Corneal topography aftercorneal perforation
}

\author{
Mirko Jankov ${ }^{1}$ \\ José Ricardo de Abreu Reggi ${ }^{2}$ \\ Adamo Lui Netto ${ }^{3}$ \\ Sandra Cayres Naufal ${ }^{1}$ \\ Paulo Elias Corrêa Dantas ${ }^{4}$ \\ Maria Cristina Nishiwaki Dantas ${ }^{5}$
}

\section{RESUMO}

Objetivo: Estudar a topografia de córnea após perfurações oculares grau 1. Métodos: Por meio de estudo clínico transversal controlado, foram realizados exames de topografia computadorizada de córnea (topógrafo EyeTech CT-2000) em ambos os olhos em 21 pacientes que haviam sido atendidos e submetidos à correção cirúrgica de perfuração corneana em um dos olhos pela equipe do Pronto Socorro do Departamento de Oftalmologia da Santa Casa de São Paulo no período de janeiro a dezembro de 1998. Os olhos contralaterais formaram o grupo controle. Resultados: Quinze pacientes $(71 \%)$ apresentaram lesões menores que $4 \mathrm{~mm}$. O astigmatismo topográfico no grupo de olhos perfurados foi $2,66 \pm 2,64 \mathrm{D}$, e $0,52 \pm 0,25$ D no grupo controle. Ao comparar distribuição dos pacientes com astigmatismo topográfico maior ou menor que 2,00 D nas categorias do tamanho médio e da configuração da lesão, observaram-se diferenças estatisticamente significativas ( $\mathrm{p}=0,04 \mathrm{e} \mathrm{p}=0,02$ respectivamente). A localização não mostrou diferença estatisticamente significativa $(\mathrm{p}=1,00)$. Não houve diferença estatisticamente significativa $(\mathrm{p}=0,98)$ entre o poder dióptrico corneal de olhos perfurados e do grupo controle. A distribuição, quanto ao padrão topográfico corneal, foi semelhante no grupo de olhos perfurados, grupo controle, bem como na literatura. Melhor acuidade visual corrigida melhor ou igual a 0,5 foi encontrado em $81 \%$ dos pacientes. Conclusões: $\mathrm{O}$ astigmatismo topográfico resultante de lesão de córnea foi maior que o do grupo controle, porém não houve mudança qualitativa do padrão topográfico destes olhos, excluindo os casos de topografia irregular. Confirma-se a correlação do astigmatismo topográfico com o tamanho da lesão, reafirmando-se o tamanho crítico de $4 \mathrm{~mm}$. Perfurações grau 1 têm bom prognóstico visual, ao passo que pior prognóstico é esperado para padrões topográficos irregulares ou lesões maiores que $4 \mathrm{~mm}$.

Descritores: Topografia da córnea; Traumatismos oculares; Ferimentos oculares

\footnotetext{
Assistente do Setor de Córnea e Doenças Externas Oculares da Santa Casa/SP.

${ }^{2}$ Chefe do Setor de Urgências Oculares da Santa Casa/ SP, Pós-graduando, Universidade de São Paulo.

${ }^{3}$ Chefe do Setor de Refração, Lentes de Contato, Transplante de Córnea e Cirurgia Refrativa da Santa Casa/SP

${ }^{4}$ Chefe do Setor de Córnea, Doenças Externas e Cirurgia Refrativa do Hospital de Olhos de Sorocaba, Assistente do Setor de Córnea e Doenças Externas Oculares da Santa Casa/SP, Pós-graduando pela Universidade de São Paulo.

${ }^{5}$ Chefe do Setor de Doenças Externas Oculares da Santa Casa/SP, Pós-graduando pela Universidade Federal de São Paulo.

Endereço para correspondência: R. Domingos de Moraes, 2267 - São Paulo (SP) CEP 04035-000.

E-mail: mirkojankov@hotmail.com

Recebido para publicação em 09.02 .2001

Aceito para publicação em 10.10.2001
}

\section{INTRODUÇÃO}

A qualidade da superfície anterior da córnea é um importante determinador da qualidade da imagem retínica porque define a forma da interface ar-lágrima, a mais potente do sistema refrativo do olho ${ }^{(1)}$.

Lesões perfurantes acontecem quando o olho é atingido por objeto cortante, causando solução completa de continuidade nos tecidos ${ }^{(2-4)}$. Podem ser divididas em 4 grupos, pela sua extensão: grau 1 - com laceração somente da córnea, com ou sem prolapso do tecido uveal; grau 2 - associação com lesão do cristalino; grau 3 - lesões do segmento posterior; grau 4 do segmento anterior e posterior ${ }^{(5-7)}$.

O astigmatismo é a maior causa de baixa de acuidade visual nestes pacientes. Acomete aproximadamente metade dos pacientes com perfura- 
ções grau $1^{(7)}$. O principal fator que determina a presença e o valor do astigmatismo é o tamanho da lesão (as maiores que 1/3 do diâmetro da córnea, ou $4 \mathrm{~mm}$, provocam mais astigmatismo), enquanto a presença de encarceração da íris, assim como a forma e a localização da lesão, parecem não influenciar ${ }^{(7-9)}$. O meridiano do astigmatismo é definido pela localização da lesão, abaulando o eixo paralelo às suturas e aplanando o perpendicular ${ }^{(9-12)}$.

A córnea possui a capacidade de reconstituir sua superfície anterior, recuperando a integridade anatômica e funcional, mesmo depois de lacerações completas, que causam descontinuidade anatômica, aproximando-se assim da forma e do poder dióptrico anterior à perfuração ${ }^{(9-11)}$. Até o termo "memória topográfica" do jargão da engenharia mecânica foi levantado em $1994^{(10)}$ e posteriormente analisado mais profundamente descrevendo as propriedades bio-mecânicas da córnea ${ }^{(11)}$.

Há um número considerável de trabalhos na literatura mundial sobre lesões perfurantes do globo ocular e seus prognós$\operatorname{ticos}^{(2-5,7-9)}$. Todos avaliaram e analisaram perfurações de todos os graus, mas em nenhum deles foi avaliado o comportamento da superfície anterior da córnea. Em contrapartida, houve trabalhos analisando 22 casos de laceração da córnea, acompanhando a regressão do astigmatismo topográfico corneal através do estudo da topografia computadorizada da córnea no período pós-operatório ${ }^{(10-11)}$.

O objetivo deste trabalho foi estudar a topografia da córnea através de estudo clínico controlado transversal, caracterizando a topografia corneal após perfurações grau 1 comparando-a com a topografia do olho controle quanto aos seguintes parâmetros: tamanho da lesão, sua localização e configuração, poder dióptrico da córnea, padrão topográfico corneal, astigmatismo topográfico e acuidade visual final.

\section{MÉTODO}

Do total de 123 pacientes atendidos e operados no Pronto Socorro do Departamento de Oftalmologia da Santa Casa de São Paulo com perfurações oculares no período de janeiro a dezembro de 1998, 45 tinham perfurações grau 1. Destes foram incluídos neste estudo 21 pacientes, e neles realizados exames de acuidade visual e topografia computadorizada de córnea. Havia 14 pacientes de sexo masculino e 7 de sexo feminino. A idade média dos pacientes era de 20,95 $\pm 12,45$ (5-46) anos. Em 14 casos, o olho acometido foi o olho esquerdo, enquanto em 7 casos, foi o direito.

\section{Critérios de inclusão}

- Perfuração corneal monocular grau 1 necessitando sutura

- Ausência de fios de sutura no momento de exame

- Ceratometria do olho contralateral dentro dos padrões médios da população normal

- Acuidade visual do olho contralateral de 1,0 sem auxílio de correção óptica

- Tempo mínimo decorrido da perfuração e suturas de 6 meses

- Idade maior que 5 anos

\section{Critérios de exclusão}

- Intervenções cirúrgicas anteriores ou qualquer outra alteração ocular anterior

- Uso de correção óptica antes do acidente

Todos os pacientes tinham sido operados sob anestesia geral, usando-se uma técnica cirúrgica padrão ${ }^{(6,9,13)}$ pelos residentes plantonistas do serviço da Residência Médica em Oftalmologia da Santa Casa de Misericórdia de São Paulo, treinados, durante o seu curso de residência, para efetuar a operação dentro do protocolo do nosso serviço. As suturas foram feitas com pontos de Mononylon ${ }^{\circledR} 10-0$ separados, eqüidistantes $1 \mathrm{~mm}$, com aproximadamente $1,5 \mathrm{~mm}$ de comprimento, com extensão de $\pm 90 \%$ da espessura da córnea, perpendiculares às margens da lesão. O critério de sucesso da cirurgia foi a reconstituição da integridade anatômica, com mínima distorção tecidual e com ausência de vazamento aquoso pela laceração, sem sinal de Seidel com Fluoresceína $2 \%^{(6-11,13)}$. Os pacientes foram acompanhados semanalmente. Os pontos foram removidos entre 6 e 12 semanas depois da intervenção cirúrgica.

Medida de acuidade visual com tabela de Snellen e exame de topografia computadorizada de córnea (TCC) foram realizados no mesmo dia após mais de 6 meses do momento de lesão, no olho perfurado e no olho contralateral. TCC foi feito em todos os olhos pelo mesmo examinador, usando-se o topógrafo EyeTech CT-2000. Sempre foi examinado primeiro o olho direito, não se levando em consideração qual dos olhos era o lesado. Foram realizadas 3 topografias sucessivas e escolhida uma delas, cumprindo-se os seguintes critérios: menor sombra palpebral, centralização adequada e boa focalização dos anéis. Depois do término da TCC de um olho, foi realizado exame do outro olho, seguindo-se os mesmos passos e cuidados.

As lesões foram classificadas segundo a sua configuração em simples (margens lisas, retas ou curvilíneas) ou complexas (extensões arborizadas), tamanho (com valor-limite de $4 \mathrm{~mm}$ ), e a sua localização em central (se a parte mais próxima da lesão foi encontrada mais perto que $2 \mathrm{~mm}$ do eixo visual) ou periférica (mais longe que $2 \mathrm{~mm}$ do eixo visual) (Figuras 1 a 3 ). As córneas lesadas e de controle foram classificadas pelo poder dióptrico da córnea, padrões topográficos corneais e grau de astigmatismo topográfico, seguindo os critérios já descritos previamente $^{(14)}$ (Figura 4). Para comparação e análise estatística foram utilizados teste de Fischer e de Student.

\section{RESULTADOS}

A relação dos pacientes deste estudo, com suas características clínicas, encontra-se na tabela 1.

O tamanho médio das lesões foi de 3,05 $\pm 2,14 \mathrm{~mm}(0,6-$ $7,6)$. Quinze pacientes $(71 \%)$ apresentaram lesões menores ou iguais a $4 \mathrm{~mm}$, enquanto 6 pacientes (29\%) maiores que $4 \mathrm{~mm}$. Treze pacientes $(62 \%)$ tinham lesão periférica e 8 pacientes (38\%), lesões centrais. Quanto à configuração, a simples foi encontrada em 17 pacientes ( $81 \%$ ) e a complexa em 4 pacientes $(19 \%)$ (Tabela 2 - Total vertical). 


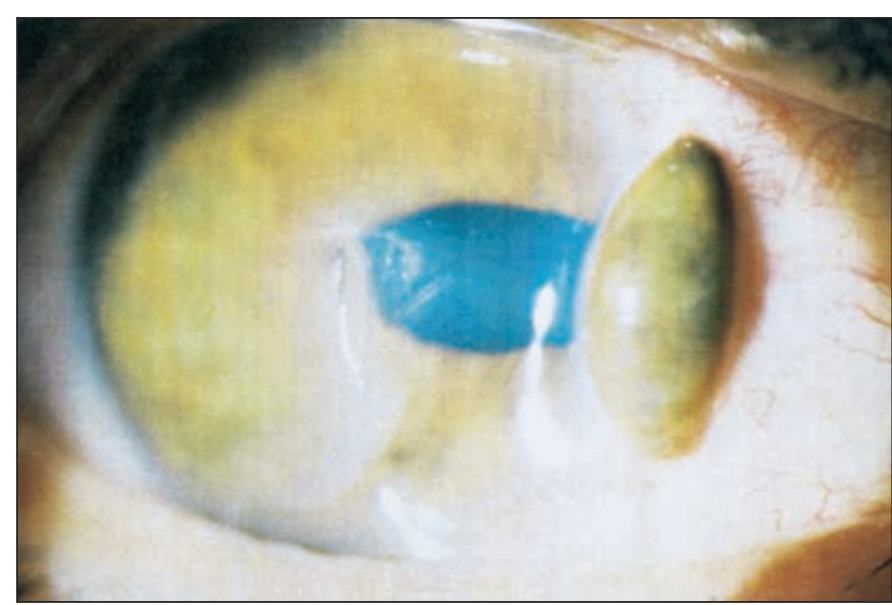

Figura 1 - Exemplo de perfurações oculares grau 1: lesão simples, periférica, 3,1 $\mathrm{mm}$ de tamanho, com prolapso de íris, ainda não suturada - paciente no 3 (Santa Casa de São Paulo, janeiro a dezembro de 1998)

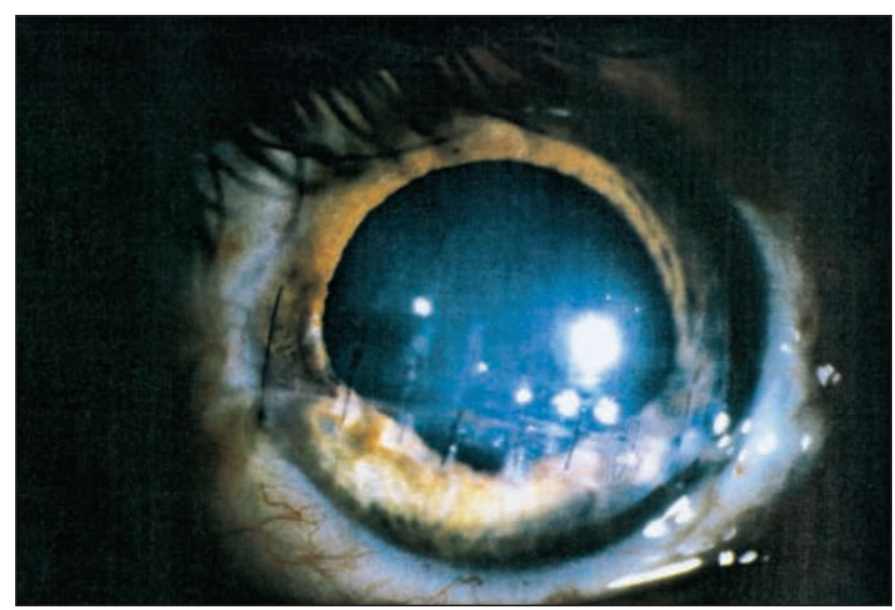

Figura 2 - Exemplo de perfurações oculares grau 1: lesão simples, periférica, $7,6 \mathrm{~mm}$ de tamanho, 3 meses após as suturas - paciente no 12 (Santa Casa de São Paulo, janeiro a dezembro de 1998)

Astigmatismo topográfico médio no grupo de olhos perfurados foi de 2,66 $\pm 2,64 \mathrm{D}(0,22-10,96)$. Doze pacientes $(57 \%)$ tinham astigmatismo topográfico médio menor ou igual a 2,00 D, enquanto 9 pacientes (43\%) maior que 2,00 D (Tabela 2 - Total horizontal) e esse foi o critério utilizado para distribuição dos pacientes em cada uma das categorias (tamanho, localização e configuração). No grupo de controle foi encontrado astigmatismo topográfico médio de $0,52 \pm 0,25 \mathrm{D}(0,11-1,00)$. Usando-se o teste de Fisher para analisar o tamanho médio da lesão entre o grupo com astigmatismo topográfico maior que 2,00 D e o com astigmatismo menor, observou-se diferença estatisticamente significativa $(\mathrm{p}=0,04)$. Analisando-se a distribuição dos pacientes com astigmatismo maior ou menor que 2,00 D dentro da categoria de configuração da lesão, também foi encontrada diferença estatisticamente significante $(\mathrm{p}=0,02)$. Em contrapartida, na categoria de localização da lesão a distribuição não mostrou diferença estatisticamente significativa $(\mathrm{p}=1,00)$.

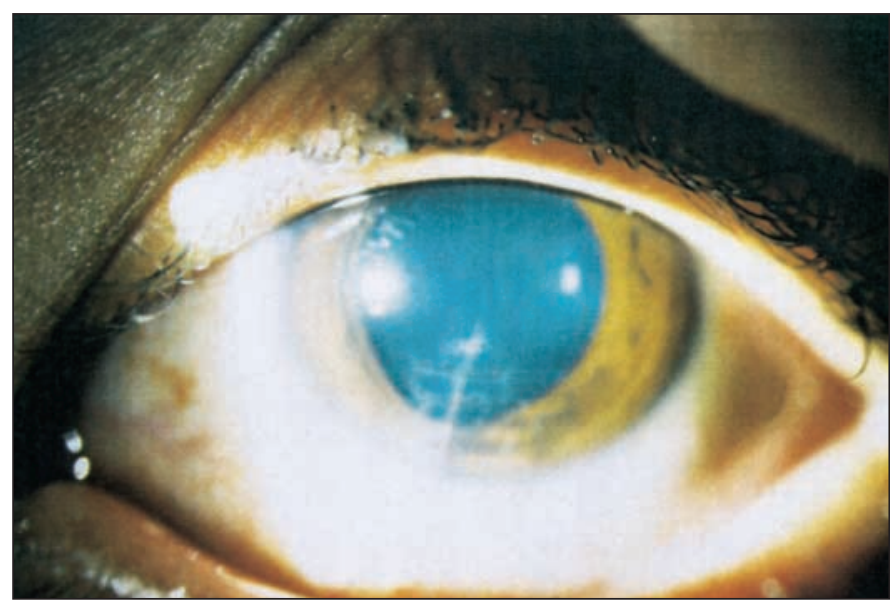

Figura 3 - Exemplo de perfurações oculares grau 1: lesão simples, central, $3,4 \mathrm{~mm}$ de tamanho, 4 meses após as suturas - paciente $\mathrm{n} \times 17$ (Santa Casa de São Paulo, janeiro a dezembro de 1998)

Poder dióptrico corneal de 43,05 $\pm 2,41 \mathrm{D}(37,82-46,28)$ foi encontrado no grupo de olhos perfurados, enquanto no grupo controle foi de 43,03 $\pm 1,94 \mathrm{D}(39,32-45,57)$. Foram realizados dois testes de análise estatística: análise de variância e t de Student. Os dois testes mostraram que não havia diferença estatisticamente significativa entre os dois grupos ( $\mathrm{p}=0,98 \mathrm{e}$ $\mathrm{p}=0,51$, respectivamente).

Quanto ao padrão topográfico corneal, três pacientes $(13,29 \%)$ tinham padrão "redondo" tanto no grupo de olhos perfurados quanto no de controle, enquanto padrão "oval" foi encontrado em 5 pacientes $(23,81 \%)$ do grupo de olhos perfurados contra $6(28,57 \%)$ do grupo controle. O padrão "borboleta simétrica" foi encontrada em 5 pacientes $(23,81 \%)$ nos dois grupos, e ao mesmo tempo "borboleta assimétrica" foi descrita em 4 pacientes $(19,05 \%)$ do grupo de olhos perfurados e em 7 pacientes $(33,33 \%)$ do grupo controle. O padrão "irregular" foi visto em 4 pacientes $(19,05 \%)$ no grupo de olhos perfurados, mas em nenhum dos pacientes do grupo controle. A relação da distribuição dos pacientes pelos padrões topográficos encontra-se no Gráfico 1.

Melhor acuidade visual corrigida (MAVC) melhor ou igual a 0,5 foi encontrado em 17 pacientes (81\%) Tabela 1 .

\section{COMENTÁRIOS}

O trabalho ideal para avaliação e acompanhamento da topografia da córnea seria um estudo prospectivo, fazendo-se TCC no mesmo olho antes e depois da lesão, antes do tratamento com pontos corneais, no período pós-operatório recente e, em seguida, periodicamente, até a retirada dos pontos, a última medida depois que a reparação corneal já se completou. Todas deveriam ser lesões exclusivamente corneais, submetidos ao tratamento padronizado e excluindo-se pacientes com outras intervenções cirúrgicas anteriores ou quaisquer outras alterações oculares anteriores. Até a presente data não foi publicado nenhum trabalho que satisfaça todos esses critérios. 


\begin{tabular}{|c|c|c|c|c|c|c|c|c|c|c|c|c|c|c|}
\hline \multirow{3}{*}{\multicolumn{2}{|c|}{$\begin{array}{l}\text { Idade } \\
\text { [anos] }\end{array}$}} & \multirow[b]{3}{*}{ Sexo } & \multirow{2}{*}{\multicolumn{4}{|c|}{ Perfuração }} & \multicolumn{6}{|c|}{ Topografia computadorizada } & \multirow{2}{*}{\multicolumn{2}{|c|}{ AV }} \\
\hline & & & & & & & \multicolumn{2}{|c|}{$\begin{array}{c}\text { Poder dióptrico corneal } \\
\text { - em dioptrias }[\mathrm{D}] \\
\text { ( ) = média aritmética }\end{array}$} & \multicolumn{2}{|c|}{$\begin{array}{c}\text { Padrão } \\
\text { topográfico }\end{array}$} & \multicolumn{2}{|c|}{$\begin{array}{c}\text { Astigmatismo } \\
\text { [D] }\end{array}$} & & \\
\hline & & & Olho & $\begin{array}{l}\text { Tamanho } \\
{[\mathrm{mm}]}\end{array}$ & $\begin{array}{l}\text { Locali- } \\
\text { zação }\end{array}$ & $\begin{array}{l}\text { Confi- } \\
\text { guração }\end{array}$ & $\begin{array}{c}\text { Olho } \\
\text { perfurado }\end{array}$ & $\begin{array}{c}\text { Olho } \\
\text { controle }\end{array}$ & $\begin{array}{c}\text { Olho } \\
\text { perfurado }\end{array}$ & $\begin{array}{c}\text { Olho } \\
\text { controle }\end{array}$ & $\begin{array}{c}\text { Olho } \\
\text { perfurado }\end{array}$ & $\begin{array}{c}\text { Olho } \\
\text { controle }\end{array}$ & SC & $\mathbf{C C}$ \\
\hline 1. & 23 & M & $\mathrm{D}$ & 1,2 & $\mathrm{P}$ & $S$ & $\begin{array}{c}43,48 / 42,45 \\
(42,97)\end{array}$ & $\begin{array}{c}42,92 / 42,13 \\
(42,53)\end{array}$ & BA & $\mathrm{BA}$ & 1,03 & 0,80 & 0,8 & 1,0 \\
\hline 2. & 17 & M & $E$ & 0,6 & C & S & $\begin{array}{c}42,66 / 42,34 \\
(42,40)\end{array}$ & $\begin{array}{c}42,88 / 42,70 \\
(42,79)\end{array}$ & $\mathrm{O}$ & $\mathrm{O}$ & 0,32 & 0,18 & 0,9 & 1,0 \\
\hline 3. & 34 & M & $E$ & 3,1 & $\mathrm{P}$ & Co & $\begin{array}{c}44,52 / 40,41 \\
(42,50)\end{array}$ & $\begin{array}{c}42,34 / 42,88 \\
(42,60)\end{array}$ & BS & BA & 4,11 & 0,54 & 0,3 & 0,9 \\
\hline 4. & 30 & M & $E$ & 1,6 & $\mathrm{P}$ & S & $\begin{array}{c}44,23 / 42,85 \\
(43,54)\end{array}$ & $\begin{array}{c}44,08 / 43,42 \\
(43,75)\end{array}$ & BA & BS & 1,38 & 0,66 & 0,7 & 1,0 \\
\hline 5. & 46 & $\mathrm{M}$ & D & 5,7 & C & $S$ & $\begin{array}{c}43,29 / 42,34 \\
(42,82)\end{array}$ & $\begin{array}{c}41,22 / 41,04 \\
(41,13)\end{array}$ & BS & $\mathrm{O}$ & 0,97 & 0,18 & 0,15 & 0,3 \\
\hline 6. & 24 & $\mathrm{M}$ & $E$ & 0,7 & C & $S$ & $\begin{array}{c}45,84 / 44,40 \\
(45,12)\end{array}$ & $\begin{array}{c}45,89 / 44,98 \\
(45,45)\end{array}$ & BS & BA & 1,44 & 0,91 & 0,8 & 1,0 \\
\hline 7. & 5 & $M$ & $E$ & 6,4 & $\mathrm{P}$ & $S$ & $\begin{array}{c}48,76 / 43,80 \\
(46,28)\end{array}$ & $\begin{array}{c}41,21 / 40,79 \\
(41,00)\end{array}$ & I & 0 & 4,96 & 0,42 & 0,15 & 0,4 \\
\hline 8. & 38 & $M$ & $E$ & 5,8 & C & Co & $\begin{array}{c}47,08 / 39,57 \\
(43,33)\end{array}$ & $\begin{array}{c}42,31 / 42,87 \\
(42,59)\end{array}$ & I & BA & 7,51 & 0,56 & $<0,1$ & 0,1 \\
\hline 9. & 10 & $\mathrm{~F}$ & $E$ & 2,8 & $\mathrm{P}$ & $S$ & $\begin{array}{c}43,44 / 45,35 \\
(44,39)\end{array}$ & $\begin{array}{c}43,74 / 43,41 \\
(43,91)\end{array}$ & 0 & BS & 1,91 & 0,33 & 0,7 & 0,9 \\
\hline 10. & 8 & $F$ & D & 2,0 & $P$ & $S$ & $\begin{array}{c}40,12 / 35,51 \\
(37,82)\end{array}$ & $\begin{array}{c}41,22 / 41,86 \\
(41,54)\end{array}$ & BA & BA & 4,61 & 0,64 & 0,3 & 0,9 \\
\hline 11. & 13 & $M$ & $E$ & 1,9 & $\mathrm{P}$ & $S$ & $\begin{array}{c}44,35 / 42,93 \\
(43,14)\end{array}$ & $\begin{array}{c}43,23 / 42,85 \\
(43,04)\end{array}$ & BS & BS & 1,42 & 0,38 & 0,7 & 1,0 \\
\hline 12. & 27 & $F$ & D & 7,6 & $P$ & $S$ & $\begin{array}{c}45,38 / 34,46 \\
(39,92)\end{array}$ & $\begin{array}{c}42,30 / 42,86 \\
(42,58)\end{array}$ & I & $\mathrm{R}$ & 10,92 & 0,56 & $<0,1$ & 0,4 \\
\hline 13. & 40 & $\mathrm{~F}$ & $E$ & 3,1 & $\mathrm{P}$ & $S$ & $\begin{array}{c}45,17 / 43,73 \\
(44,45)\end{array}$ & $\begin{array}{c}45,64 / 45,09 \\
(45,37)\end{array}$ & $\mathrm{R}$ & BS & 1,44 & 0,56 & 0,8 & 0,9 \\
\hline 14. & 7 & M & D & 0,9 & C & $S$ & $\begin{array}{c}39,91 / 37,72 \\
(38,81)\end{array}$ & $\begin{array}{c}40,16 / 39,16 \\
(39,66)\end{array}$ & 0 & 0 & 2,18 & 1,0 & 0,6 & 0,7 \\
\hline 15. & 26 & $M$ & $E$ & 4,8 & $\mathrm{P}$ & $S$ & $\begin{array}{c}45,36 / 42,89 \\
(44,14)\end{array}$ & $\begin{array}{c}44,50 / 43,81 \\
(44,16)\end{array}$ & BS & BS & 2,47 & 0,69 & 0,7 & 1,0 \\
\hline 16. & 5 & $\mathrm{~F}$ & $E$ & 1,3 & C & Co & $\begin{array}{c}40,22 / 37,68 \\
(38,95)\end{array}$ & $\begin{array}{c}39,73 / 38,90 \\
(39,32)\end{array}$ & I & BA & 2,54 & 0,83 & 0,3 & 0,5 \\
\hline 17. & 10 & $\mathrm{~F}$ & $E$ & 3,4 & C & $S$ & $\begin{array}{c}45,69 / 45,09 \\
(45,39)\end{array}$ & $\begin{array}{c}45,84 / 45,30 \\
(45,57)\end{array}$ & $\mathrm{R}$ & $\mathrm{R}$ & 0,60 & 0,54 & 1,0 & 1,0 \\
\hline 18. & 18 & $F$ & D & 0,7 & $\mathrm{P}$ & $S$ & $\begin{array}{c}42,00 / 41,78 \\
(41,89)\end{array}$ & $\begin{array}{c}44,72 / 44,61 \\
(44,67)\end{array}$ & $\mathrm{R}$ & $\mathrm{R}$ & 0,22 & 0,11 & 0,9 & 1,0 \\
\hline 19. & 8 & $M$ & D & 5,5 & $P$ & Co & $\begin{array}{c}47,76 / 43,80 \\
(45,78)\end{array}$ & $\begin{array}{c}41,22 / 40,80 \\
(41,01)\end{array}$ & 0 & 0 & 3,96 & 0,42 & 0,2 & 0,7 \\
\hline 20. & 19 & $M$ & $E$ & 1,4 & C & $S$ & $\begin{array}{c}45,89 / 44,71 \\
(45,40)\end{array}$ & $\begin{array}{c}45,64 / 45,34 \\
(45,49)\end{array}$ & BA & BA & 1,18 & 0,30 & 0,4 & 0,8 \\
\hline 21. & 32 & $M$ & $E$ & 3,6 & $\mathrm{P}$ & $S$ & $\begin{array}{c}45,39 / 44,74 \\
(45,06)\end{array}$ & $\begin{array}{c}45,54 / 45,34 \\
(45,44)\end{array}$ & 0 & $\mathrm{O}$ & 0,66 & 0,20 & 1,0 & 1,0 \\
\hline
\end{tabular}

No nosso estudo, de 123 pacientes com perfurações oculares, atendidos e operados no nosso Departamento durante o ano de 1998, quarenta e cinco pacientes (37\%) sofreram perfurações grau 1 , o que está de acordo com $41 \%$ dos pacientes da literatura $^{(7)}$. Desse grupo, dezesseis pacientes foram excluí- dos: 11 deles tinham AV sem correção óptica do olho contralateral pior que 1,0 , três foram submetidos à operação de transplante de córnea tectônico e em 2 pacientes a lesão foi bilateral. Portanto, apenas 29 pacientes satisfizeram todos os requisitos para nosso estudo. Vinte e três desses pacientes foram 

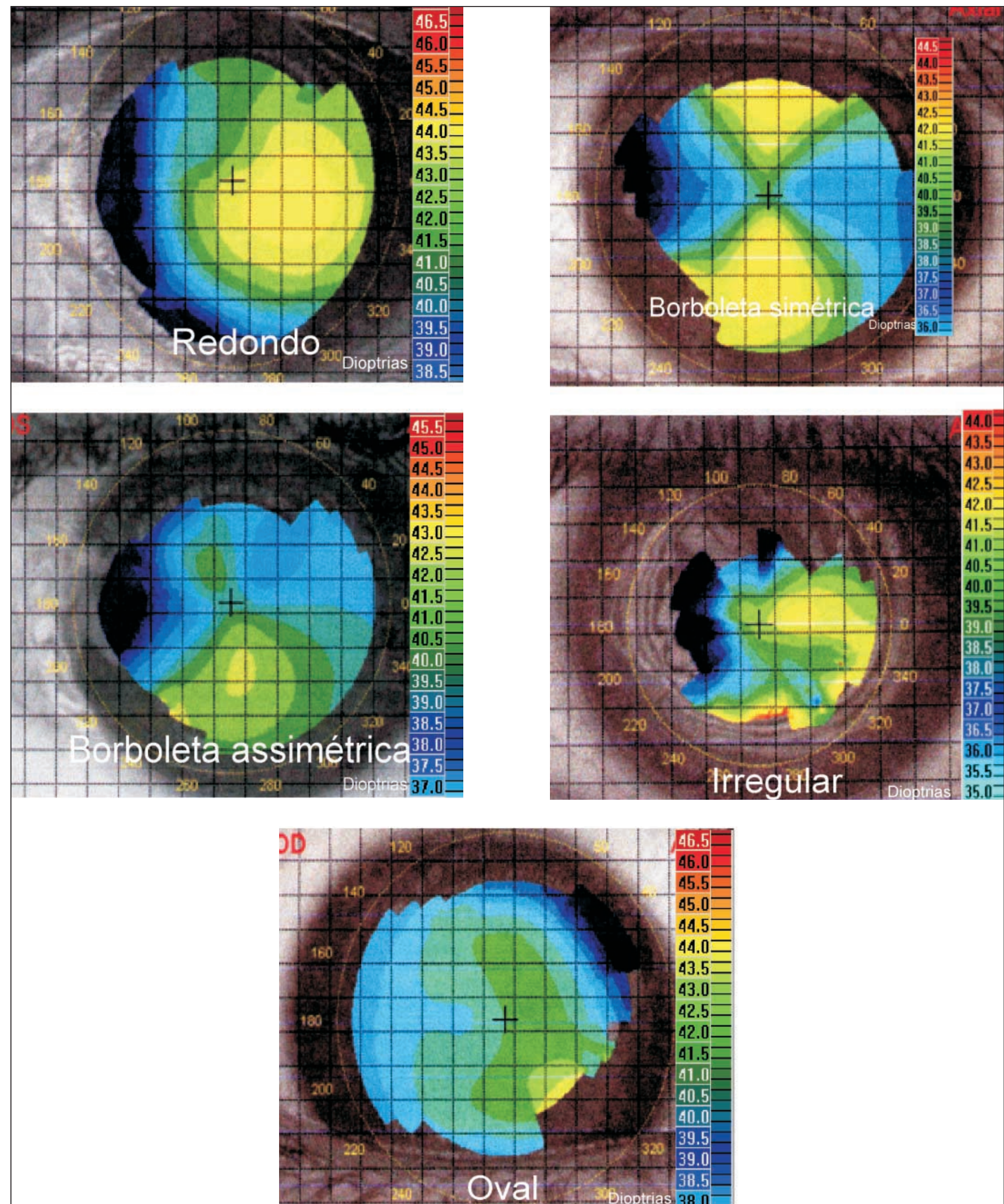

Figura 4 - Padrões topográficos corneais dos olhos perfurados (pacientes com perfurações oculares grau 1, Santa Casa de São Paulo, no período de janeiro a dezembro de 1998) 
localizados, dos quais 21 fizeram parte deste estudo. Curiosamente, os dois pacientes que se recusaram a ser re-examinados alegaram que estavam satisfeitos com a sua qualidade de visão.

Um dos maiores problemas para a realização deste trabalho foi o estabelecimento do grupo controle. Idealmente, o grupo controle deveria ser o olho perfurado antes da lesão. Como a TCC não é realizada de rotina, foi impossível obter essas informações para serem usadas como grupo controle. Optamos por usar o olho contralateral como grupo controle.

A nossa decisão foi feita levando-se em consideração os vários trabalhos que mostram que a média de poder dióptrico

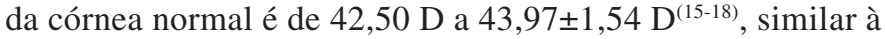
encontrada na nossa amostra no olho controle de 43,03 $\pm 1,94$ D. Além disto, os trabalhos que mostram a igualdade ${ }^{(19)}$ ou semelhança do poder dióptrico corneal ${ }^{(16)}$ entre os dois olhos, fortaleceram a nossa argumentação.

Acrescente-se o fato de que os próprios critérios de inclusão e exclusão visaram escolher somente os pacientes com a acuidade visual, antes da perfuração, de 1,0 sem uso de correção óptica, em ambos os olhos. O astigmatismo topográfico do grupo controle foi de 0,52 $\pm 0,25(0,11-1,00) \mathrm{D}$. Desta forma, acreditamos que conseguimos selecionar, da maneira melhor possível, os pacientes nos quais os olhos contralaterais poderiam ser incluídos no grupo controle, mesmo sem a TCC do mesmo olho, anterior à perfuração.

No estudo mais completo da literatura foram analisados 228 pacientes com todos os graus de perfuração, sendo de maior prevalência as perfurações grau 1 (41\%). Foi demonstrado que aproximadamente metade desses pacientes tinha astigmatismo corneal, sendo o tamanho da lesão o seu principal fator provocante; lesões acima de 1/3 de diâmetro corneal provocaram significativamente mais astigmatismo topográfico do que as menores que esse limite ${ }^{(7)}$. Nesse estudo, porém, não foi feita a comparação com o outro olho, impossibilitando a conclusão da restituição da topografia corneal.
Um outro trabalho envolvendo 122 pacientes com lesões córneo-esclerais, com ou sem envolvimento do segmento posterior (graus 1, 2 e 4 pela classificação Eagling et al.) foi publicado $^{(8)}$. Quanto ao astigmatismo topográfico no grupo de pacientes que corresponderia à perfuração grau 1 (somente 21 pacientes), o tamanho da lesão mostrou-se também como principal fator na sua magnitude, definindo-se como valor limite de lesão de 4 mm.

A distribuição do tipo de padrão topográfico nos olhos normais e nos perfurados, pelos principais estudos da literatu$\mathrm{ra}^{(11,16,18,20)}$, está exposta na tabela 3. A superfície anterior da córnea normal não é uniforme, apesar de ser possível classificá-la, usando TCC, nos seguintes padrões topográficos: "redondo", "oval", "borboleta simétrica", "borboleta assimétrica" e "irregular". Pode-se observar que a distribuição é relativamente homogênea, com uma preponderância leve do padrão "borboleta assimétrica", presente em aproximadamente um terço da população normal, e menor incidência do padrão "irregular" $(14,16,18,20)$. No nosso trabalho, a distribuição foi semelhante à do grupo controle (Gráfico 1) e à dos olhos normais (Tabela 3), confirmando a constatação sobre a semelhança da distribuição entre os olhos perfurados e os olhos normais.

Vários trabalhos foram escritos analisando e discutindo os fatores que influenciam o tamanho de astigmatismo final nas perfurações corneais ${ }^{(9-10,13)}$, dentre eles os principais se encontram na Tabela $4^{(7-8,11)}$. Todos os autores concordaram que o fator principal é o tamanho da lesão, ao fazer a análise do tamanho crítico que provoca astigmatismo clinicamente significativo, i.e. maior que 2,00 D. Foi afirmado que poucas lesões do tamanho menor ou igual a $1 / 3$ de diâmetro corneal ( $4 \mathrm{~mm}$ ) provocariam astigmatismo significativo ( 7 e $27 \%$ respectivamente), comparado com $67 \%$ e $51 \%$, das lacerações maiores que este limite com o mesmo astigmatismo ${ }^{(7-8)}$. No outro estudo foi confirmada essa distribuição nos casos das lesões pequenas, porém constatou-se que somente $25 \%$ das lesões maiores provocaram o astigmatismo maior que $2,00 \mathrm{D}^{(11)}$. Uma ex-
Olhos perfurados

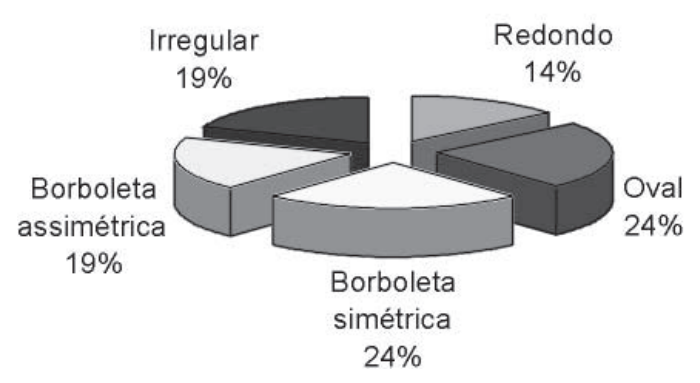

Olhos normais

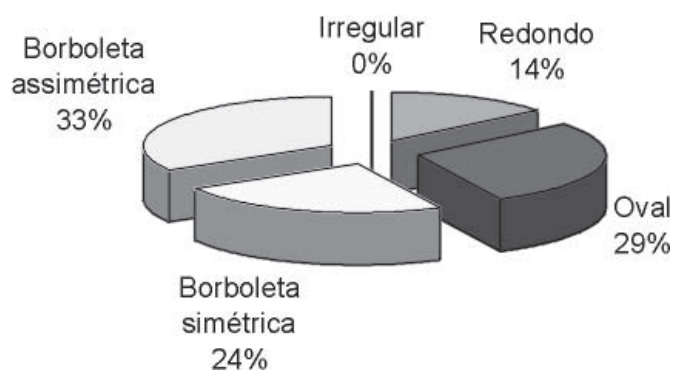

Gráfico 1 - Distribuição do tipo de padrão topográfico corneal das lesões nos pacientes com perfuraç̃es oculares grau 1, atendidos e operados no Pronto Socorro do Departamento de Oftalmologia da Santa Casa de São Paulo, no período de janeiro a dezembro de 1998 
plicação para tal discordância poderia ser o fato de que o tamanho limite utilizado foi de $5 \mathrm{~mm}$, o que resultaria numa distribuição diferente quanto ao astigmatismo topográfico final.

No nosso trabalho, $27 \%$ das lesões menores ou iguais a $4 \mathrm{~mm}$ provocaram astigmatismo topográfico clinicamente significativo, comparado com $73 \%$ dos maiores que $4 \mathrm{~mm}$, o que está de acordo com os encontrados na literatura (Tabela 4).

Usando-se o teste de Fisher, ao comparar o tamanho médio e a configuração da lesão, ao astigmatismo topográfico maior ou menor que 2,00 D, observamos diferenças estatisticamente significativas ( $\mathrm{p}=0,04$ e $\mathrm{p}=0,02$ respectivamente). A localização, portanto, comparada ao astigmatismo topográfico maior ou menor que 2,00 D, não mostrou diferença estatisticamente significativa $(\mathrm{p}=1,00)$. A partir desses dados, pode-se confirmar, o já constatado nos outros trabalhos, que o tamanho da lesão tem um papel importante no astigmatismo final, enquanto a localização da lesão não tem influência estatisticamente significativa. $\mathrm{O}$ resultado do teste de Fisher, quanto à configuração da lesão, pode ser explicado pelo fato de que, levando-se em consideração o mecanismo de lesão, a participação das lesões de configuração simples nas perfurações grau 1, é esperadamente maior do que a das lesões complexas ${ }^{(7-8,13)}$

Nenhum dos trabalhos mencionados até agora analisou aspecto topográfico da córnea, mas somente acuidade visual, junto ao valor absoluto de astigmatismo, foram avaliados para comparação. Um único trabalho foi publicado na literatura sobre o aspecto topográfico da córnea após perfurações corneais em 22 olhos $^{(11)}$. Acompanhando os pacientes, fez TCC no período pós-operatório após 2 e 14 semanas, concluindo

\begin{tabular}{|c|c|c|c|}
\hline $\begin{array}{l}\text { abela 2. Astigmatismo } \\
\text { onfiguração das lesões } \\
\text { rau 1, atendidos e operac } \\
\text { fttalmologia da Santa Ca }\end{array}$ & $\begin{array}{l}\text { os pacier } \\
\text { s no Pron } \\
\text { de São } \\
\text { embro d }\end{array}$ & ${ }_{8}^{\text {no perí }}$ & $\begin{array}{l}\text { tamento de } \\
\text { janeiro a }\end{array}$ \\
\hline & Astig & latismo - olho & s perfurados \\
\hline Laceração & $\begin{array}{c}\leq 2,00 \mathrm{D} \\
\text { Número(\%) }\end{array}$ & $\begin{array}{c}>2,00 \mathrm{D} \\
\text { Número(\%) }\end{array}$ & $\begin{array}{c}\text { Total } \\
\text { Número(\%) }\end{array}$ \\
\hline Tamanho & $11(52,38)$ & $4(19,05)$ & $15(71,43)$ \\
\hline$>4$ & & & \\
\hline $\begin{array}{cc}\text { Localização } & \begin{array}{c}\text { Central } \\
\text { Periférica }\end{array}\end{array}$ & $\begin{array}{ll}5 & (23,81) \\
7 & (33,33)\end{array}$ & $\begin{array}{l}3(14,29) \\
6(28,57)\end{array}$ & $\begin{array}{c}8(38,10) \\
13(61,91)\end{array}$ \\
\hline Configuração Simples & $12(57,14)$ & $5(23,81)$ & $17(80,95)$ \\
\hline Complex & $0(0)$ & & \\
\hline Total & $12(57,14)$ & $9(42,86)$ & $21(100)$ \\
\hline
\end{tabular}

que padrões topográficos dos olhos perfurados são semelhantes aos dos olhos normais. Chegou à conclusão, também, de que a curvatura da córnea, e conseqüentemente poder dióptrico, se restitui depois de lacerações corneais, atingindo os valores médios de curvaturas anteriores ao acidente, introduzindo o termo "memória topográfica".

Apesar da metodologia correta e do número aparentemente considerável de pacientes, somente 7 deles realmente tinham perfuração grau 1. Os outros (graus 2 ou 4) foram efetuadas outras intervenções cirúrgicas subseqüentes (facectomia com ou sem implante de lente intra-ocular ${ }^{(21)}$, vitrectomia via pars plana, ou "scleral buckling"), alterando assim significativamente, os contornos da superfície anterior da córnea, tornando qualquer conclusão estatística sobre a topografia da córnea inviável e não confiável, devido ao pequeno tamanho da amostra e o número grande de variáveis de confusão.

Comparando o valor do astigmatismo topográfico antes e depois da retirada dos fios de sutura os autores afirmaram que existe redução significativa do astigmatismo topográfico corneal. Outro fato é que valor do poder dióptrico corneal do grupo dos olhos perfurados se mostrou igual ao do grupo controle (42,4 D). O desvio padrão, porém, no grupo dos olhos perfurados foi maior do que o encontrado no grupo controle.

No nosso estudo, o poder dióptrico corneal do grupo dos olhos perfurados comparado ao do grupo controle não mostrou diferença estatisticamente significativa, confirmando a tese de que a córnea é capaz de recuperar a grande parte da sua integridade anatômica e funcional. O poder dióptrico corneal dos dois grupos, encontra-se dentro da média previamente descrita na literatura. Além disto, há semelhança entre a distribuição dos padrões topográficos do grupo dos olhos perfurados e do grupo controle (Gráfico 1), bem como à das córneas normais (Tabela 3).

Analisando-se padrão topográfico corneal caso a caso observa-se que em 10 pacientes $(47,62 \%)$ houve diferença de padrão entre grupo dos olhos perfurados e grupo controle. Porém, tendo em vista as limitações de vídeo-topografia nas córneas irregulares (4 pacientes com padrão topográfico 'irregular') e a grande semelhança de padrões 'borboleta simétrica' e 'borboleta assimétrica' (3 pacientes), sobraram somente 3 pacientes $(14,29 \%)$ apresentando 'mudança' do padrão topográfico entre dois grupos. A alteração do padrão topográfico nesses pacientes $(5,9$ e 13$)$ pode ser explicada pelos gran-

\begin{tabular}{|c|c|c|c|c|c|c|}
\hline \multirow[b]{2}{*}{$\begin{array}{l}\text { Padrão topográfico } \\
\text { corneal }\end{array}$} & \multicolumn{4}{|c|}{ Olhos normais } & \multicolumn{2}{|c|}{ Olhos perfurados } \\
\hline & $\begin{array}{l}\text { BOGAN } \\
(1990)[\%]\end{array}$ & $\begin{array}{c}\text { BECHARA } \\
(1996)[\%]\end{array}$ & $\begin{array}{l}\text { KANPOLAT } \\
(1997)[\%]\end{array}$ & $\begin{array}{l}\text { JANKOV } \\
(1999)[\%]\end{array}$ & $\begin{array}{l}\text { NAVON } \\
(1997)[\%]\end{array}$ & $\begin{array}{l}\text { JANKOV } \\
(2000)[\%]\end{array}$ \\
\hline Redondo & 22,6 & 17,3 & 14,0 & 14,3 & & 14,3 \\
\hline Oval & 20,8 & 25,6 & 11,4 & 28,6 & 36,0 & 23,8 \\
\hline Borboleta simétrica & 17,5 & 24,1 & 29,0 & 23,8 & 27,0 & 23,8 \\
\hline Borboleta assimétrica & 32,1 & 33,0 & 33,3 & 33,3 & 23,0 & 19,1 \\
\hline Irregular & 7,1 & 0 & 12,3 & 0 & 14,0 & 19,1 \\
\hline
\end{tabular}




\begin{tabular}{|c|c|c|c|c|c|c|c|c|c|}
\hline \multirow{3}{*}{\multicolumn{2}{|c|}{ Laceração }} & \multirow{2}{*}{\multicolumn{2}{|c|}{ EAGLING (1976)[\%] }} & \multicolumn{4}{|c|}{ Astigmatismo [D] } & \multirow{2}{*}{\multicolumn{2}{|c|}{ JANKOV (2000)[\%] }} \\
\hline & & & & \multicolumn{2}{|c|}{ BARR (1983)[\%] ${ }^{\#}$} & \multicolumn{2}{|c|}{ NAVON (1997)[\%] ${ }^{\#}$} & & \\
\hline & & $\leq 2,00$ & $>2,00$ & $\leq 2,00$ & $>2,00$ & $\leq 2,00$ & $>2,00$ & \multicolumn{2}{|c|}{$\leq 2,00 \quad>2,00$} \\
\hline \multirow[t]{2}{*}{ Tamanho } & $\leq 4 \mathrm{~mm}$ & $93^{*}$ & $7^{*}$ & 73 & 27 & $90^{\S}$ & $10^{\S}$ & 73 & 27 \\
\hline & $>4 \mathrm{~mm}$ & $33^{*}$ & $67^{*}$ & 49 & 51 & $75^{\S}$ & $25^{\S}$ & 17 & 83 \\
\hline \multirow[t]{2}{*}{ Localização } & Central & - & - & - & - & 67 & 33 & 63 & 37 \\
\hline & Periférica & - & - & - & - & 92 & 8 & 54 & 46 \\
\hline \multirow[t]{2}{*}{ Configuração } & Simples & - & - & - & - & 81 & 19 & 71 & 29 \\
\hline & Complexa & - & - & - & - & 83 & 17 & 0 & 100 \\
\hline
\end{tabular}

des tamanhos de lesão que, apesar de nem todos serem menores que $4 \mathrm{~mm}$, mostraram-se suficientes para mudarem o padrão topográfico nesses olhos.

A diminuição da qualidade de visão, avaliada pela diminuição de acuidade visual, é, de fato, a conseqüência mais importante de qualquer lesão ocular. No nosso estudo foi confirmado o bom prognóstico de perfurações oculares grau 1, com $86 \%$ dos pacientes enxergando melhor ou igual a $0,5 \mathrm{com}$ correção óptica, concordando, assim, com os $94 \%$ dos pacientes no estudo da literatura ${ }^{(7)}$. Em contraste, em outro traba1 ho ${ }^{(8)}$ foi relatada AV melhor ou igual a 0,5 somente em $62 \%$ dos pacientes, porém não ficou claro se essa foi alcançada com a melhor correção óptica, ou sem tal correção. O restante dos trabalhos da literatura sobre o tema ${ }^{(1,3-5)}$ não apresentaram resultados discriminando graus de lesão, o que os tornou inconclusivos quanto à correlação entre o grau de perfuração e a acuidade visual final.

Dentre os pacientes com MAVC pior que 0,5 (pacientes número $5,7,8$ e 12) observou-se que em todos eles (100\%) o tamanho da lesão foi maior que $4 \mathrm{~mm}$. Além disso, $75 \%$ dos casos mostrou padrão topográfico irregular, enquanto somente um paciente tinha padrão "borboleta simétrica". Estes dados mostram que os casos com tamanho da lesão maior que 4 $\mathrm{mm}$, bem como tendo padrão topográfico "irregular", têm pior prognóstico visual.

\section{CONCLUSÕES}

- O poder dióptrico corneal médio do grupo das córneas perfuradas, comparado ao poder dióptrico corneal médio do grupo controle no nosso estudo, bem como ao dos olhos normais da literatura, não demonstrou diferença estatisticamente significativa.

- O astigmatismo topográfico resultante de lesão de córnea foi maior que do grupo controle, porém não houve mudança qualitativa do padrão topográfico destes olhos, excluindo os casos de topografia irregular.

- Perfurações grau 1 tem bom prognóstico visual, tendo MAVC melhor ou igual a 0,5 em $86 \%$ dos pacientes.

- Observou-se pior prognóstico visual (MAVC pior que $0,5)$ em padrões topográficos irregulares ou lesões maiores que $4 \mathrm{~mm}$.
- Confirmou-se a correlação entre o valor do astigmatismo topográfico final e o tamanho da lesão, reafirmando o tamanho-crítico de $4 \mathrm{~mm}$.

- Não existiu correlação entre o valor do astigmatismo topográfico final e a localização da lesão.

\section{ABSTRACT}

Purpose: To analyze the corneal topography after grade I ocular perforating injuries. Methods: Twenty-one patients, attended and operated at the Department of Ophthalmology of Santa Casa de Sao Paulo during the year 1998, were included into this controlled clinical transversal study. Computerized corneal topography was performed using the EyeTech CT2000 Corneal Topographer. Fellow eyes formed the control group. Results: Fifteen patients (71\%) had wounds less than $4 \mathrm{~mm}$. The mean corneal topographic astigmatism was $2.66 \pm$ $2.64 \mathrm{D}$ in the group of perforated eyes, and $0.52 \pm 0.25 \mathrm{D}$ in the control group. Statistically significant differences were found comparing the distribution of the patients with topographic corneal astigmatism higher or lower than $2.00 \mathrm{D}$ in the categories of medium corneal wound size $(\mathrm{p}=0.04)$ and wound conformation $(\mathrm{p}=0.02)$. Localization showed no statistical difference $(\mathrm{p}=1.00)$. No statistically significant difference $(\mathrm{p}=0.98)$ was found comparing the mean corneal power in the group of perforated eyes with the control group. The final distribution of the topographic corneal pattern showed similarity between the group of perforated eyes and the control group, as well as that of the literature data. Bestcorrected visual acuity better or equal to 0.5 was found in $81 \%$ of the patients. Conclusions: The topographic astigmatism resulting from corneal laceration was larger than that in the control group, although there was no qualitative change concerning topographic pattern in these eyes, having excluded the cases with irregular topography. Correlation between the final astigmatism and the wound size was found, confirming the critical size of $4 \mathrm{~mm}$. Grade I ocular perforating injuries show good visual outcome, which is not the case with irregular topography or wounds larger than $4 \mathrm{~mm}$.

Keywords: Corneal topography; Eye injuries; Cornea/injuries 


\section{REFERÊNCIAS}

1. Maloney RK, Bogan SJ, Waring GO. Determination of corneal image-forming properties from corneal topography. Am J Ophthalmol 1993;115:31-41.

2. Alves MR, Kara José N, Prado Jr J, Usuba FS, Onclinx TM, Marantes CR. Ferimento perfurante ocular: 400 casos admitidos na Clínica Oftalmológica do Hospital das Clínicas da Faculdade de Medicina da Universidade de São Paulo. Arq Bras Oftalmol 1995;58:342-5.

3. Bonanomi MTBC, Alves MR, Kara José N, Souza Jr NA. Ferimento perfurante do globo ocular em adultos. Arq Bras Oftalmol 1980;43:71-7.

4. Kara José N, Alves MR, de Oliveira PR. Como educar a população para a prevenção do trauma ocular. Arq Bras Oftalmol 1992;55:160-2.

5. Kara José N, Alves MR, Bonanomi MTBC, Souza Jr NA. Ferimento perfurante de globo ocular na infância. Rev Bras Oftalmol 1981;40:243-54.

6. Moraes NSB, Motta M, Melamed J. Ferimentos oculares. In: Terapêutica clínica ocular. São Paulo: Roca; 1995. p.211-8.

7. Eagling EM. Perforating injuries of the eye. Br J Ophthalmol 1976;60:732-6.

8. Barr CC. Prognostic factors in corneoscleral lacerations. Arch Ophthalmol 1983;101:919-24.

9. Rowsey JJ, Hays JC. Refractive reconstruction for acute eye injuries. Ophthalmic Surg 1984;15:569-74.

10. Smith SD, Navon SE. Computerized topography of a full-thickness corneal laceration. Ophthalmic Surg 1994;25:630-2.

11. Navon SE. Topography after repair of full-thickness corneal laceration. J Cataract Refract Surg 1997;23:495-501.
12. Strelow S, Cohen EJ, Leavitt KG, Laibson PR. Corneal topography for selective suture removal after penetrating keratoplasty. Am J Ophthalmol 1991;112:657-65.

13. Shingleton BJ, Hersh P, Kenyon KR. Eye trauma. St. Louis: Mosby Year Book; 1991.

14. Bogan SJ, Waring III GO, Ibrahim O, Drews C, Curtis L. Classification of normal corneal topography based on computer-assisted videokeratography. Arch Ophthalmol 1990;108:945-9.

15. Rowsey JJ, Reynolds AE, Brown R. Corneal topography - corneascope. Arch Ophthalmol 1981;99:1093-1100.

16. Dingeldein SA, Klyce SD. The topography of normal corneas. Arch Ophthalmol 1989;107:512-8.

17. Ninn-Pedersen K, Stenevi U, Ehinger B. Cataract patients in a defined swedish population 1986-1990 II. Preoperative observations. Acta Ophthalmol (Copenh) 1994;72:10-5.

18. Kanpolat A, Simsek T, Alp NM. The evaluation of normal corneal topography in emmetropic eyes with computer-assisted videokeratography. CLAO J 1997;23:168-71.

19. Longanesi L, Cavallini GM, Toni R. Quantitative clinical anatomy of the human cornea in vivo. A morphometric study by ultrasonic pachymetry and computer-assisted viedokeratroscopy. Acta Anat (Basel) 1996;157:73-9.

20. Bechara SJ, Onclinx TM, Marantes CR, Avakian A, Rotemberg M, Kara Jose N. Padrões topográficos em olhos normais com baixo astigmatismo [resumo]. Arq Bras Oftalmol 1996;59:390.

21. Werblin TP. Refractive stability after cataract extraction using a 6.5-milimeter scleral pocket Incision with horizontal or radial sutures. J Refract Corneal Surg 1994;10:339-42.

\title{
XIII Congresso da Sociedade Norte-Nordeste de Oftalmologia
}

\section{5 a 18 de Maio de 2002 Maceió - AL}

\author{
Informações: tel.: (82) 327-6859 \\ fax: (82) 231-1619 \\ e-mail: nnoftalmo.maceio@globo.com
}

